\title{
Role of MALAT1 in gynecological cancers: Pathologic and therapeutic aspects (Review)
}

\author{
FENG-HUA QIAO ${ }^{1}$, MIN TU² and HONG-YAN LIU ${ }^{3}$ \\ Departments of ${ }^{1}$ Gynecology and ${ }^{2}$ Orthopedics, Second People's Hospital of Jingmen; \\ ${ }^{3}$ Department of Gynecology, Maternal and Child Health Hospital of Jingmen, Jingmen, Hubei 448000, P.R. China
}

Received June 28, 2020; Accepted February 2, 2021

DOI: $10.3892 / 01.2021 .12594$

\begin{abstract}
Gynecological cancers, including breast, ovarian, uterine, vaginal, cervical and vulvar cancers are among the major threats to modern life, particularly to female health. Long non-coding RNAs (lncRNAs) play critical roles in normal development of organisms, as well as the tumorigenesis process, and metastasis-associated lung adenocarcinoma transcript 1 (MALAT1) is a large infrequently spliced lncRNA, which have been implicated in different gynecological cancers. MALAT1 is overexpressed in breast, ovarian, cervical and endometrial cancers, which initiates cancer progression by inducing changes in the expression of several anti-apoptotic and epithelial-to-mesenchymal transition-related genes. Targeting MALAT1 is an important strategy to combat gynecological cancers, and application of RNA-interference technology and chemotherapeutic process are crucial to target and minimize MALAT1 activity. The present review discusses the role of MALAT1 in gynecological cancers, and potential strategies to target this lncRNA to develop cancer therapeutics. However, further clinical studies are required to determine the prognostic potential of MALAT1 in gynecological cancers.
\end{abstract}

\section{Contents}

1. Introduction

2. Long non-coding RNA and cancer

3. MALAT1: Structure and function

4. Pathogenic role of MALAT1 in gynecological cancers

Correspondence to: Dr Min Tu, Department of Orthopedics, Second People's Hospital of Jingmen, 39 Xiangshan Road, Jingmen, Hubei 448000, P.R. China

E-mail: tm1981@jcut.edu.cn

Dr Hong-Yan Liu, Department of Gynecology, Maternal and Child Health Hospital of Jingmen, 8 Xiangshan 2nd Road, Jingmen, Hubei 448000, P.R. China

E-mail: liuhy_jnh@yahoo.com

Key words: breast cancer, cervical cancer, long non-coding RNA, MALAT1, RNA-interference, ovarian cancer
5. Targeting MALAT1 in gynecological cancer therapeutics

6. Future research

7. Conclusions

\section{Introduction}

Gynecologic cancer is any cancer of the female reproductive organs, including ovarian, uterine, vaginal, cervical and vulvar cancers. Generally, all women are at risk of developing gynecological cancers, and risk increases with age (1). Each gynecologic cancer is unique, with different signs and symptoms, different risk factors and different therapeutic strategies (2,3). Gynecological cancers are associated with high mortality rates worldwide as it is difficult to detect the cancers in early stage (1). Gynecological cancers are among the major threats to modern life, particularly to female health (1-3). An essential part of the gynecologic assessment is examination of the breasts. Breast cancer is recognized more often by gynecologists than any other physician (4). Women with breast cancer are at risk of developing a second primary gynecologic cancer, particularly uterine and ovarian cancers (4).

Breast cancer is the most common invasive cancer in women that develops from breast tissues. It is the second most common cancer among women and the leading cause of cancer-associated mortality, accounting for approximately 500,000 mortalities per year worldwide (5). It is a multifactorial disease affected by several risk factors, including age, sex, genetics, ethnicity, environmental factors, high levels of certain hormones, including estrogen and progesterone, lifestyle and diet (5-8). Ovarian cancer is a cancerous growth that begins in the ovaries (9). It is caused by several factors, including age, genetic mutations, metabolic abnormalities, endometriosis and hormone replacement therapy (9-12). Cervical cancer is a cancer arising from the cervix (the entrance to the womb from the vagina), which predominantly affects sexually active women aged $30-45$ years. Infection with the human papilloma virus (HPV) is considered a major risk factor for cervical cancer $(13,14)$. Other risk factors for this disease include smoking, a weak immune system, birth control pills, early age at first intercourse, multiple sex partners and low socioeconomic status (13-15). Endometrial cancer is a common gynecological cancer that begins in the uterus (16). Several factors may increase the risk of endometrial cancer, including 
age, obesity, hormone therapy, genetic mutations, hypertension, diabetes mellitus and favorable prognosis (16-18). Other types of gynecological cancers include vaginal cancer, which forms in the tissues of the vagina, and vulvar cancer, which forms in the external genital organ of women. However, these types of gynecological cancers are rare, and thus infrequently studies (2). Similar to cervical cancer, both vaginal and vulvar cancers are associated with HPV infection $(2,19)$.

\section{Long non-coding RNA and cancer}

The human genome has been estimated to contain 23,000 long non-coding RNA (lncRNA) genes, which are more abundant than 20,000 protein-coding genes (20). IncRNA genes are an important population of non-coding RNAs, without protein-coding capacity $(20,21)$. IncRNAs transcripts are $>200$ nucleotides (nt) in length, and represent a stringent cell-type/tissue specificity (22). IncRNAs play critical roles in normal development of organisms, as well as the tumorigenesis process $(22,23)$. The crucial roles of lncRNAs include dosage compensation, imprinting, chromatin rearrangement, histone modification, modification of alternative splicing genes, as well as gene expression (23). According to their functions and expression patterns in tumor cells/tissues, IncRNAs can be classified as tumor suppressor genes or oncogenes $(24,25)$. IncRNAs play different roles in the regulation of cancer-related pathways, such as the Wnt, Hedgehog, Notch and PI3K/AKT/mTOR pathways, and regulate the plasticity of cancer stem cells (26). IncRNAs interact with microRNAs (miRNAs/miRs), mRNAs, proteins and genomic DNA to exert their physiological and pathological functions $(26,27)$. Deregulation of several 1ncRNAs has been detected in different types of cancer, such as, breast, ovarian, cervical and prostate cancer, which suggests the use of lncRNAs as markers for cancer detection and prognosis, as well therapeutic targets for cancer treatment $(22,28)$.

\section{MALAT1: Structure and function}

Metastasis-associated lung adenocarcinoma transcript 1 (MALAT1) is a large and infrequently spliced lncRNA, also known as nuclear enriched abundant transcript 2, HCN, LINC00047, NCRN00047 and PRO2853 (29). MALAT1 is abundant in several human cell types, with the highest expression levels in pancreas and lung cells $(29,30)$. MALAT1 is a single-exon gene, located within human chromosome 11q13 and mouse chromosome $19 \mathrm{qA}$. The primary structure of MALAT1 contains $\sim 8 \mathrm{~kb}$ in humans and $\sim 7 \mathrm{~kb}$ in mice $(31,32)$. Its 3 '-end lacks a poly (A) tail structure and can be processed by RNase $\mathrm{P}$ and RNase $\mathrm{Z}$ cleavage into a long 6.7-kb transcript, which yields an additional 3'-short tRNA-like ncRNA and a 5'-long MALAT1-associated small cytoplasmic RNA (31-33). Following transcription, the longer form of MALAT1 is retained in the nucleus and specifically localizes to nuclear speckles. These structures of MALAT1 are enriched in pre-mRNA splicing factors, and serve as storage and assembly/modification sites. MALAT1 may interact with the serine/arginine-rich family of splicing factors, which affects the distribution of splicing factors in nuclear speckle domains, and regulates tissue- or cell-type specific alternative splicing in a phosphorylation-dependent manner (31,34). MALAT1 acts as an activator of gene expression by mediating the interaction with the demethylated form of chromobox homolog 4, also known as polycomb 2, a component of the Polycomb Repressive Complex $1(31,34,35)$.

MALAT1 has also been reported to regulate several pathological processes, ranging from diabetes complications to cancer (36). MALAT1 was initially identified as a factor associated with high metastatic potential and poor prognosis in stage I non-small cell lung cancer (NSCLC) $(36,37)$. MALAT1 is overexpressed in different types of tumors, including lung, liver, gastric, pancreatic, renal, colon, bladder, breast, bone cancers and gynecological cancers (38). MALAT1 regulates the expression of metastasis-associated genes and cell motility via transcriptional and/or post-transcriptional regulation $(38,39)$. It has been demonstrated that various upstream regulators may bind to the promoter to activate the transcription of MALAT1, resulting in upregulated MALAT1 expression in different types of cancer (40). Sp1 is associated with lung cancer, whereas JMJD1A is associated with neuroblastoma, the sex determining region Y-box (Sox) 17 is associated with esophageal cancer, and TDP-43 is associated with NSCLC $(40,41)$. Serine/arginine-rich splicing factor 1 (SRSF1) is associated with breast cancer, while yes-associated protein and SRSF1 are associated with liver cancer $(40,41)$. Previous studies have identified the common genetic variants (single nucleotide polymorphisms) of MALAT1 associated with the risk of different types of cancer (42-44). Thus, MALAT1 plays an important factor in carcinogenesis. The present review discusses the expression patterns of MALAT1 in breast cancer and gynecological cancers, in the perspectives of carcinogenesis and therapeutics.

\section{Pathogenic role of MALAT1 in gynecological cancers}

Fig. 1 and Table I present the pathogenic role of MALAT1 in gynecological cancers. Breast cancer exosomes promote cancer cell proliferation by modulating exosomal MALAT1 regulation. MALAT1 is considered a proinflammatory factor, which regulates the lipopolysaccharide (LPS)-induced inflammatory response by interacting with nuclear factor kappa-light-chain-enhancer of activated B cells (NF- $\kappa \mathrm{B})$ of breast cancer cells $(39,45)$. $N F-\kappa B$ also plays an important role in regulating the epithelial-to-mesenchymal transition (EMT) process, and promotes matrix metalloproteinase (MMP)-9 and vimentin expression by binding to the promoter of vimentin $(39,45)$. Previous studies have demonstrated that MALAT1 expression is upregulated in breast cancer cells and tissues (46-49). Gomes et al (50) reported that an antisense transcript of MALAT1, named TALAM1, mediates MALAT1 response in human breast cancer. They reported that MALAT1 locus is spanned by TALAM1, and downregulation of TALAM1 induces breast cancer cell aggressiveness and malignancy. In addition, Wu et al (51) observed the highest level of MALAT1 expression in metastatic triple-negative breast cancer and trastuzumab-resistant human epidermal growth factor receptor 2 (HER2) overexpressing (HER2+) cells. They demonstrated that upregulated MALAT1 expression via activation of the PI3K/AKT pathway induced EMT-like phenotypes and cell invasiveness in HER2+ cells (51). Huang et al (52) 
Table I. Molecular mechanisms of MALAT1 in gynecological cancers.

\begin{tabular}{|c|c|c|c|}
\hline Cancer type & Mechanism & Effects & (Refs.) \\
\hline \multirow[t]{3}{*}{ Breast cancer } & Downregulates TALAM1 expression & Induces cell aggressiveness and malignancy & $(50)$ \\
\hline & Activates the PI3K/AKT pathway & $\begin{array}{l}\text { Induces EMT-like phenotype and cell } \\
\text { invasiveness }\end{array}$ & $(51)$ \\
\hline & Downregulates microRNA-145 expression & $\begin{array}{l}\text { Enhances proliferation, migration and tube } \\
\text { formation }\end{array}$ & $(52)$ \\
\hline \multirow[t]{4}{*}{ Ovarian cancer } & $\begin{array}{l}\text { Activates the JAK2/STAT3 signaling } \\
\text { pathway }\end{array}$ & $\begin{array}{l}\text { Promotes proliferation and inhibits cell } \\
\text { apoptosis }\end{array}$ & $(59)$ \\
\hline & $\begin{array}{l}\text { Activates the } \mathrm{PI} 3 \mathrm{~K} / \mathrm{AKT} \text { and } \mathrm{Wnt} / \beta \text {-catenin } \\
\text { pathways }\end{array}$ & Induces EMT-like phenotype & $(62)$ \\
\hline & $\begin{array}{l}\text { Influences HUVECs and stimulates } \\
\text { angiogenesis related genes }\end{array}$ & Promotes angiogenesis & $(65)$ \\
\hline & $\begin{array}{l}\text { Upregulates MMP13 expression and } \\
\text { downregulates MMP19 and ADAMTS1 } \\
\text { expression }\end{array}$ & Induces cell migration and invasion & $(66)$ \\
\hline \multirow[t]{2}{*}{ Cervical cancer } & Activates the PI3K/AKT pathway & Induces EMT-like phenotype & $(71)$ \\
\hline & Activates AKT/mTOR & $\begin{array}{l}\text { Increases cell viability, cell migration and } \\
\text { invasion, and EMT }\end{array}$ & $(70)$ \\
\hline \multirow{2}{*}{$\begin{array}{l}\text { Endometrial } \\
\text { cancer }\end{array}$} & Activates the $\mathrm{Wnt} / \beta$-catenin pathway & Induces cell migration and invasion & $(72)$ \\
\hline & MALAT1 rs664589 C>G polymorphism & Associated with cancer risk & $(73)$ \\
\hline
\end{tabular}

MALAT1, metastasis-associated lung adenocarcinoma transcript 1; EMT, epithelial-to-mesenchymal transition; HUVECs; human umbilical vein endothelial cells; MMP, matrix metalloproteinase.

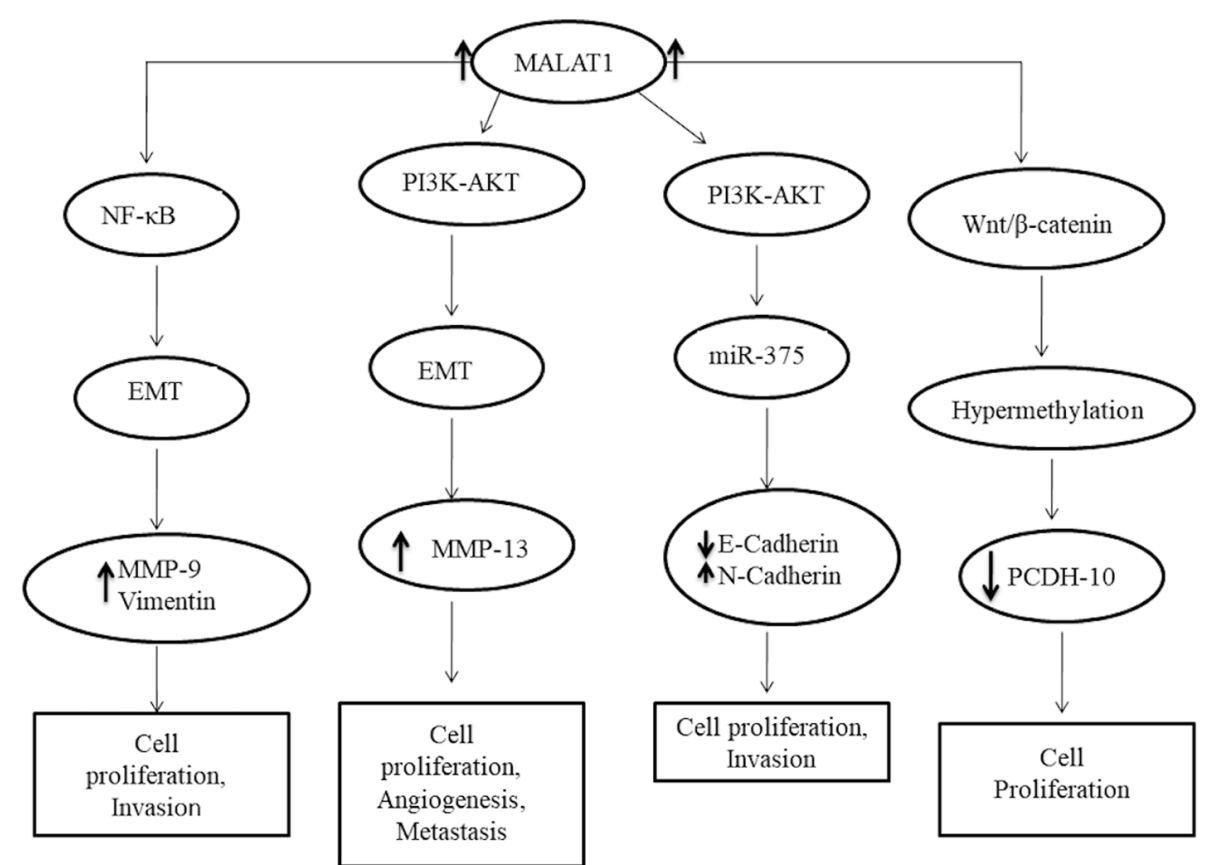

Figure 1. Pathological role of MALAT1 in gynecological cancers. Overexpression of MALAT1 promotes tumor initiation in gynecological cancers by inducing changes in the expression of several anti-apoptotic and EMT-related genes. MALAT1, metastasis-associated lung adenocarcinoma transcript 1; EMT, epithelial-to-mesenchymal transition; NF- $\mathrm{BB}$, nuclear factor kappa-light-chain-enhancer of activated B cells; MMP, matrix metalloproteinase; miR, microRNA; PCDH10, protocadherin 10 .

reported an interaction between MALAT1 and miR-145, and observed that upregulated MALAT1 expression significantly enhanced the proliferation, migration and tube formation of MCF-7 cells, whereas miR-145 expression inversely changed in breast cancer tissues. Kim et al (53) demonstrated contradictory effects of MALAT1 in breast cancer cell proliferation and invasion. They reported that MALAT1 acts as a metastasis-suppressing IncRNA rather than a metastasis promoter 
Table II. Major strategies in cancer therapeutics by targeting MALAT1.

\section{Strategies}

Inactivation of MALAT1 by small RNA technologies

Development of MALAT1-specific chemotherapeutic drugs Inactivation of MALAT1 by applying inhibitors of MALAT1 Degradation of MALAT1 by using microRNA response elements

Epigenetic modification of MALAT1 gene to silence its expression

MALAT1, metastasis-associated lung adenocarcinoma transcript 1.

in breast cancer. Furthermore, overexpression of MALAT1 was demonstrated to suppress breast cancer cell migration and invasion by binding and inactivating the pro-metastatic transcription factor, TEAD (53). Studies by Kwok et al (54) and Eastlack et al (55) have reported that MALAT1 can act as a tumor suppressor in breast cancer. As these findings contradict the role of MALAT1 as an important therapeutic target for breast cancer, it was suggested that MALAT1 should be thoroughly investigated to determine its dual roles across different types of cancer (56). Peng et al (42) demonstrated the association of MALAT1 rs619586 A $>\mathrm{G}$ polymorphism with a reduced risk of breast cancer in the Chinese Han population.

Several studies have suggested that MALAT1 is highly expressed in ovarian cancer tissues and cell lines. Overexpression of MALAT1 promotes tumor initiation in ovarian cancer by inducing EMT $(57,58)$. For example, Sun et al (59) demonstrated that overexpression of MALAT1 promotes proliferation and suppresses the apoptosis of ovarian cancer cells by activating the JAK2/STAT3 signaling pathway. EMT maintains the mesenchymal cell phenotype and plays an essential role in cancer cell migration and invasion through E-cadherin, N-cadherin, vimentin and EMT-inducing transcription factors, such as Twist, Snail, Slug and Zeb $(60,61)$. MALAT1 may influence EMT in ovarian cancer by activating the PI3K/AKT pathway (62). Jin et al (62) reported that MALAT1 regulates ovarian cancer cell proliferation, migration and apoptosis via the Wnt/ $\beta$-catenin signaling pathway. Wnt is a cell growth factor that promotes Frizzled receptor expression and activates the scaffolding protein, Dishevelled, to induce intracellular signal transduction and glycogen synthase-kinase $3 \beta$ phosphorylation. $\beta$-catenin (a subunit of the cadherin protein complex) protein binds to E-cadherin to increase the adhesion between cells (63). $\beta$-catenin accumulates in the nucleus, which activates the transcription of the downstream cyclin D1 and other target genes to increase the cell proliferation, tumor formation and cell migratory and invasive abilities $(63,64)$. Qiu et al $(65)$ reported that MALAT1 can be transferred from ovarian cancer cells to recipient human umbilical vein endothelial cells (HUVECs) via exosomes. They indicated that the metastatic ovarian cancer cells promote angiogenesis by transferring exosomal MALAT1 to recipient HUVECs, which in turn, triggers HUVECs to promote angiogenesis by stimulating angiogenesis-related genes, including vascular endothelial growth factor (VEGF)-A, VEGF-D, epithelial neutrophil-activating peptide-78, placental growth factor, interleukin-8, angiogenin, basic fibroblast growth factor and leptin. Zhou et al (66) demonstrated that the efficacy of MALAT1 in promoting ovarian cancer progression can also be mediated by upregulating MMP13 expression and downregulating MMP19 and ADAMTS1 expression.

MALAT1 expression is upregulated in cervical cancer tissues compared with normal cervix tissues (67). Jiang et al (68) demonstrated that MALAT1 expression is upregulated in HPV-positive cervical squamous cells, which suggests that high MALAT1 expression is associated with HPV in cervical cancer. Upregulated MALAT1 expression promotes the proliferation and invasion, and decreases the apoptosis of cervical cancer cells (67). MALAT1 can act as an oncogene by sponging miRNA in HR-HPV positive cervical cancer cells (69). MALAT1 is involved in regulating the EMT process in cervical cancer (70). Wang et al (71) reported that overexpressing MALAT1 significantly increases BRWD1 mRNA expression by activating the PI3K/AKT pathway in HeLa and C-33A cells. Han et al (70) demonstrated that overexpression of periostin in cervical cells is positively associated with MALAT1 expression and negatively associated with miR-202-3p expression. Furthermore, it was suggested that the MALAT1/miR-202-3p/periostin axis increases cell viability, cell migration and invasion, and EMT in HeLa or SiHa cells by activating the AKT/mTOR signaling pathway (70).

Increased levels of MALAT1 have been detected in endometrial cancer. Zhao et al (72) identified MALAT1 as a direct transcriptional target of $\mathrm{Wnt} / \beta$-catenin in endometrial cancer cells. The protocadherin 10 (PCDH10) is a novel Wnt pathway regulatory element that acts as a tumor suppressor. In endometrial cancer, PCDH10 expression is downregulated by promoter hypermethylation, which induces aberrant activation of the Wnt/ $\beta$-catenin signaling pathway via the direct binding site of TCF4 in MALAT1 promoter region (72). Chen et al (73) demonstrated the association of MALAT1 rs664589 $\mathrm{C}>\mathrm{G}$ polymorphism with the risk of endometrial cancer in Southern Chinese women. It was demonstrated that individuals with CGG haplotypes have a higher risk of developing endometrial cancer compared with the wild-type GCG haplotype carriers (73). Collectively, these findings suggest the potential role of MALAT1 in inducing the development of different gynecological cancers.

\section{Targeting MALAT1 in gynecological cancer therapeutics}

The treatment of gynecologic cancer depends on several factors, including the type, stage and grade, as well as the general health of the patient. The main treatment options for gynecologic cancer include surgery, chemotherapy, radiation therapy and hormone therapy, either alone or in a combination with each other $(2,58)$. Despite advancements in modern multimodality chemotherapeutic strategies, there is a high chance of local relapse and tumor metastasis $(2,58)$. Thus, targeted therapies are used to target both metastatic progressions and decrease the risk of recurrence in the treatment of gynecologic cancer (2). Targeting MALAT1 is an important strategy to combat gynecological cancers. The important strategies to target MALAT1 are summarized in Table II. 
Several studies have reported that high MALAT1 expression is associated with increased stage, recurrence and decreased survival in gynecological cancers $(56,66,67)$. Thus, suppressing MALAT1 expression may be a novel target for the treatment of gynecological cancers. MALAT1 knockdown via small interfering RNA (siRNA) impairs the exosomemediated pro-angiogenic activity of HUVECs through certain key angiogenesis-related genes, and significantly decreases cancer cell proliferation, migration and invasion $(74,75)$.

Li et al (39) performed RNA reverse transcription-associated trap sequencing and demonstrated that MALAT1 bound to the promoter regulatory element of the translation elongation factor $1-\alpha 1$ gene (EEF1 $\alpha 1)$. Knockdown of MALAT1 significantly downregulated EEF1 $\alpha 1$ expression, which in turn decreased cell proliferation and invasion by arresting cells at the $\mathrm{G}_{0} / \mathrm{G}_{1}$ phase in breast cancer cells (39). Liu et al (75) reported that miR-1 functions as a tumor suppressor by targeting K-RAS and MALAT1. They found that downregulated hsa-miR-1 expression in breast cancer tissues and restoration of miR-1 in breast cancer cells inhibits tumor growth and cell migration and invasion, and increases apoptosis (75). Knockdown of endogenous MALAT1 using MALAT1 short hairpin RNA (shRNA) significantly increases miR-145 expression and can inhibit proliferation, migration and tube formation by decreasing VEGF expression in breast cancer cells (52). Arun et al (76) reported that genetic loss or systemic MALAT1 knockdown using antisense oligonucleotides (ASOs) in the MMTV (mouse mammary tumor virus)-PyMT mouse mammary carcinoma model decreased tumor growth and metastatic capacity (76). In addition, MALAT1 knockdown in 4T1 xenograft mice significantly decreased the inflammatory responses by decreasing tumor necrosis factor- $\alpha$, and weakened tumor metastasis of lung induced by LPS (39).

Gordon et al (77) reported that MALAT1 promotes ovarian cancer progression by regulating RBFOX2-mediated alternative splicing. It was demonstrated that suppression of MALAT1 decreased the proliferation, invasion, anchorage independent growth, and increased anoikis in multiple anoikis-resistant ovarian cancer cell lines by decreasing RBFOX2 expression and EMT-related genes (77). Guo et al (63) demonstrated that downregulating MALAT1 expression inhibits cell proliferation, invasion and migration, arrests cell cycle progression in the $\mathrm{S}$ phase and induces cell apoptosis in ovarian cancer cell lines by inhibiting activation of the Wnt/ $\beta$-catenin signaling pathway. Bai et al (78) reported that MALAT1 knockdown significantly attenuates cisplatin resistance and induces apoptosis in cisplatin-resistant ovarian cancer cells by inhibiting the Notch1 signaling pathway. Several studies have performed lentivirus-mediated artificial miRNA interference to determine the effect of MALAT1 in ovarian cancer cells $(66,79)$. It has been reported that miR-200c is negatively associated with MALAT1 expression (80). Furthermore, MALAT1 knockdown suppresses the viability, and the invasive and migratory abilities of ovarian cancer cells (80). MALAT1 knockdown may also suppress tumor growth via miR-506-dependent regulation (81). MALAT1 knockdown decreases MMP13 protein expression, while increasing MMP19 and ADAMTS1 expression, resulting in $\mathrm{G}_{0} / \mathrm{G}_{1}$ cell cycle arrest and apoptosis in ovarian cancer cell lines (66).
Silencing MALAT1 expression via shRNA decreases cervical cancer cell viability, induces cell apoptosis, represses the cell invasive capacity, increases $G_{1}$ phase cells and decreases $S$ phase or $\mathrm{G}_{2} / \mathrm{M}$ ratio (82). Overexpression of several miRNAs, such as, miR-1, miR-145, miR-506 and miR-200c or the use of MALAT1 siRNA decreases the cell invasive and migratory abilities, downregulates mesenchymal markers, $\beta$-catenin and Vimentin, and upregulates E-cadherin expression $(52,75,81,83)$. Xia et al (84) used metformin for type 2 diabetes, to assess its effects on the migratory and invasive abilities of human cervical cancer cells, and it was demonstrated that metformin markedly inhibits the proliferation and angiogenesis of human cervical cancer cells and cervical cancer cell xenografts in nude mice (84).

It has been reported that miR-200 family members are enriched in endometrial cancer, while MALAT1 is expressed at low levels. Li et al (85) used a xenograft tumor model to demonstrate that targeting the miR-200c/MALAT1 axis inhibits endometrial cancer cell proliferation and EMT-associated protein expression in vivo. Thus, MALAT1 may act as a key target in therapeutic research on gynecological cancers.

\section{Future research}

Gynecological cancers are among the most common causes of mortality in women worldwide as it is difficult to detect the cancer in early stage (2). Dysregulation of MALAT1 has been reported in gynecological cancers, and thus may be used as a potential therapeutic target (86-88). Several studies have reported on the interference approaches of MALAT1 knockdown by RNA $(78,81)$. However, MALAT1 is less accessible than mRNAs to siRNAs as it is in the nucleus. Direct targeting of MALAT1 through RNA interference is not possible as this technology is not yet feasible in routine clinical practice. ASOs are regarded as a valuable approach to antagonize MALAT1. Systemic knockdown of MALAT1 using ASOs may provide an exciting prospective avenue for investigating the use of MALAT1 ASOs in a therapeutic setting to decrease tumor progression. ASOs are considered more advantageous over siRNAs due to their higher specificity and fewer off-target effects, as well as their independency on the RNA-induced silencing complex machinery. The limitations of ASOs include off-target toxicity effects, low affinity for the target, high vulnerability to degradation and poor delivery to the target tissues. Chemical modifications may overcome some of these limitations of ASOs by enhancing affinity, specificity and improving effective delivery to target tissues, with lower toxicity $(28,38,88,89)$. In addition, targeting MALAT1 with specific drugs or stimulating its functions in clinically feasible ways may lead to the suppression of cancer development $(28,38,88-90)$.

\section{Conclusions}

Gynecological cancers are a major threat to modern life in women. The conventional treatments of gynecological cancers remain unsatisfactory due to the adverse side effects. Early diagnosis is essential for the effective treatment of gynecological cancers. As MALAT1 expression is upregulated in different types of human cancer, including 
gynecological cancers, MALAT1 may act as a potential diagnostic marker, and may be recognized as an effective therapeutic target for gynecological cancers. However, more clinical studies are required to determine the prognostic potential of MALAT1 in gynecological cancers. A better understanding of the role of MALAT1 in tumor progression and tumor metastasis may help discover novel anti-metastatic targets for the effective treatment of gynecological cancers.

\section{Acknowledgements}

Not applicable.

\section{Funding}

The present review was supported by the Provincial Science Foundation of Hubei, China (grant no. 2020cfb702).

\section{Availability of data and materials}

Not applicable.

\section{Authors' contributions}

FHQ drafted the initial manuscript, MT and HYL conceived the present review and revised the manuscript for important intellectual content. MT and HYL confirmed the authenticity of all the raw data. All authors have read and approved the final manuscript.

\section{Ethics approval and consent to participate}

Not applicable.

\section{Patient consent for publication}

Not applicable.

\section{Competing interests}

The authors declare that they have no competing interests.

\section{References}

1. Jeong HM, Kwon MJ and Shin YK: Overexpression of cancer-associated genes via epigenetic derepression mechanisms in gynecologic cancer. Front Oncol 4: 12, 2014.

2. Zhou XM, Zhang $H$ and Han $X$ : Role of epithelial to mesenchymal transition proteins in gynecological cancers: Pathological and therapeutic perspectives. Tumour Biol 35: 9523-9530, 2014.

3. Liu DT: EMMPRIN in gynecologic cancers: Pathologic and therapeutic aspects. Tumour Biol 36: 4883-4888, 2015.

4. Gusberg SB: The gynecologist and breast cancer. Isr J Med Sci 17: 843-846, 1981.

5. Imani S, Hosseinifard $\mathrm{H}$, Cheng J, Wei $\mathrm{C}$ and $\mathrm{Fu} \mathrm{J}$ : Prognostic value of EMT-inducing transcription factors (EMT-TFs) in metastatic breast cancer: A systematic review and meta-analysis. Sci Rep 6: 28587, 2016.

6. Liu S, Wang H, Zhang L, Tang C, Jones L, Ye H, Ban L, Wang A, Liu Z, Lou F, et al: Rapid detection of genetic mutations in individual breast cancer patients by next-generation DNA sequencing. Hum Genomics 9: 2, 2015.
7. Brody JG, Rudel RA, Michels KB, Moysich KB, Bernstein L, Attfield KR and Gray S: Environmental pollutants, diet, physical activity, body size, and breast cancer: Where do we stand in research to identify opportunities for prevention? Cancer 109 (12 Suppl): S2627-S2634, 2007.

8. Rezaeian M, Sharifirad G, Mostafavi F, Moodi M and Abbasi MH: The effects of breast cancer educational intervention on knowledge and health beliefs of women 40 years and older, Isfahan, Iran. J Educ Health Promot 3: 43, 2014.

9. Tania M, Khan MA and Song Y: Association of lipid metabolism with ovarian cancer. Curr Oncol 17: 6-11, 2010.

10. Goff BA: Ovarian cancer: Screening and early detection. Obstet Gynecol Clin North Am 39: 183-194, 2012.

11. Piek JM, van Diest PJ and Verheijen RH: Ovarian carcinogenesis: An alternative hypothesis. Adv Exp Med Biol 622: 79-87, 2008.

12. Vargas-Hernández VM, Moreno-Eutimio MA, Acosta-Altamirano G and Vargas-Aguilar VM: Management of recurrent epithelial ovarian cancer. Gland Surg 3: 198-202, 2014.

13. Canavan TP and Doshi NR: Cervical cancer. Am Fam Physician 61: 1369-1376, 2000.

14. Jemal A, Bray F, Center MM, Ferlay J, Ward E and Forman D: Global cancer statistics. CA Cancer J Clin 61: 69-90, 2011.

15. Muñoz N, Castellsagué X, de González AB and Gissmann L: Chapter 1: HPV in the etiology of human cancer. Vaccine 24 (Suppl 3): S3/1-10, 2006.

16. Pessoa JN, Freitas AC, Guimaraes RA, Lima J, Dos Reis HL and Filho AC: Endometrial assessment: When is it necessary? J Clin Med Res 6: 21-25, 2014

17. Colombo N, Preti E, Landoni F, Carinelli S, Colombo A, Marini C and Sessa C; ESMO Guidelines Working Group: Endometrial cancer: ESMO clinical practice guidelines for diagnosis, treatment and follow-up. Ann Oncol 24 (Suppl 6): vi33-vi38, 2013.

18. Lozano R, Naghavi M, Foreman K, Lim S, Shibuya K, Aboyans V, Abraham J, Adair T, Aggarwal R, Ahn SY, et al: Global and regional mortality from 235 causes of death for 20 age groups in 1990 and 2010: A systematic analysis for the global burden of disease study 2010. Lancet 380: 2095-2128, 2012.

19. Dittmer C, Katalinic A, Mundhenke C, Thill M and Fischer D: Epidemiology of vulvar and vaginal cancer in Germany. Arch Gynecol Obstet 284: 169-174, 2011.

20. Kopp F and Mendell JT: Functional classification and experimental dissection of long noncoding RNAs. Cell 172: 393-407, 2018.

21. Bassett AR, Akhtar A, Barlow DP, Bird AP, Brockdorff N, Duboule D, Ephrussi A, Ferguson-Smith AC, Gingeras TR, Haerty W, et al: Considerations when investigating lncRNA function in vivo. Elife 3: e03058, 2014.

22. Soudyab M, Iranpour $M$ and Ghafouri-Fard S: The role of long non-coding RNAs in breast cancer. Arch Iran Med 19: 508-517, 2016.

23. Geisler S and Coller J: RNA in unexpected places: Long non-coding RNA functions in diverse cellular contexts. Nat Rev Mol Cell Biol 14: 699-712, 2013.

24. Grote P and Herrmann BG: Long noncoding RNAs in organogenesis: Making the difference. Trends Genet 31: 329-335, 2015.

25. Nagano $T$ and Fraser P: No-nonsense functions for long noncoding RNAs. Cell 145: 178-181, 2011.

26. Zhu Y, Luo M, Brooks M, Clouthier SG and Wicha MS: Biological and clinical significance of cancer stem cell plasticity. Clin Transl Med 3: 32, 2014.

27. Ørom UA and Shiekhattar R: Long noncoding RNAs usher in a new era in the biology of enhancers. Cell 154: 1190-1193, 2013.

28. Wahlestedt C: Targeting long non-coding RNA to therapeutically upregulate gene expression. Nat Rev Drug Discov 12: 433-446, 2013.

29. Zhang X, Hamblin MH and Yin KJ: The long noncoding RNA Malat1: Its physiological and pathophysiological functions. RNA Biol 14: 1705-1714, 2017.

30. Hutchinson JN, Ensminger AW, Clemson CM, Lynch CR, Lawrence JB and Chess A: A screen for nuclear transcripts identifies two linked noncoding RNAs associated with SC 35 splicing domains. BMC Genomics 8: 39, 2007.

31. Zhang B, Arun G, Mao YS, Lazar Z, Hung G, Bhattacharjee G, Xiao X, Booth CJ, Wu J, Zhang C and Spector DL: The lncRNA Malat1 is dispensable for mouse development but its transcription plays a cis-regulatory role in the adult. Cell Rep 2: 111-123, 2012.

32. Bernard D, Prasanth KV, Tripathi V, Colasse S, Nakamura T, Xuan Z, Zhang MQ, Sedel F, Jourdren L, Coulpier F, et al: A long nuclear-retained non-coding RNA regulates synaptogenesis by modulating gene expression. EMBO J 29: 3082-3093, 2010. 
33. Wilusz JE, Freier SM and Spector DL: 3' end processing of a long nuclear-retained noncoding RNA yields a tRNA-like cytoplasmic RNA. Cell 135: 919-932, 2008.

34. Tano K, Mizuno R, Okada T, Rakwal R, Shibato J, Masuo Y, Ijiri K and Akimitsu N: MALAT-1 enhances cell motility of lung adenocarcinoma cells by influencing the expression of motility-related genes. FEBS Lett 584: 4575-4580, 2010.

35. Yang L, Lin C, Liu W, Zhang J, Ohgi KA, Grinstein JD, Dorrestein PC and Rosenfeld MG: ncRNA- and Pc2 methylation-dependent gene relocation between nuclear structures mediates gene activation programs. Cell 147: 773-788, 2011.

36. Ji P, Diederichs S, Wang W, Böing S, Metzger R, Schneider PM, Tidow N, Brandt B, Buerger H, Bulk E, et al: MALAT-1, a novel noncoding RNA, and thymosin beta4 predict metastasis and survival in early-stage non-small cell lung cancer. Oncogene 22 8031-8041, 2003.

37. Gutschner T, Hämmerle M, Eissmann M, Hsu J, Kim Y, Hung G, Revenko A, Arun G, Stentrup M, Gross M, et al: The noncoding RNA MALAT1 is a critical regulator of the metastasis phenotype of lung cancer cells. Cancer Res 73: 1180-1189, 2013.

38. Shi X, Sun M, Wu Y, Yao Y, Liu H, Wu G, Yuan D and Song Y: Post-transcriptional regulation of long noncoding RNAs in cancer. Tumour Biol 36: 503-513, 2015.

39. Li Z, Xu L, Liu Y, Fu S, Tu J, Hu Y and Xiong Q: LncRNA MALAT1 promotes relapse of breast cancer patients with postoperative fever. Am J Transl Res 10: 3186-3197, 2018.

40. Zhao M, Wang S, Li Q, Ji Q, Guo P and Liu X: MALAT1: A long non-coding RNA highly associated with human cancers. Oncol Lett 16: 19-26, 2018

41. Tee AE, Ling D, Nelson C, Atmadibrata B, Dinger ME, Xu N, Mizukami T, Liu PY, Liu B, Cheung B, et al: The histone demethylase JMJD1A induces cell migration and invasion by up-regulating the expression of the long noncoding RNA MALAT1. Oncotarget 5: 1793-1804, 2014.

42. Peng R, Luo C, Guo Q, Cao J, Yang Q, Dong K, Wang S, Wang K and Song C: Association analyses of genetic variants in long non-coding RNA MALAT1 with breast cancer susceptibility and mRNA expression of MALAT1 in Chinese Han population. Gene 642: 241-248, 2018.

43. Chen Q, Zhu C and Jin Y: The oncogenic and tumor suppressive functions of the long noncoding RNA MALAT1: An emerging controversy. Front Genet 11: 93,2020.

44. Ni W, Wang X, Sun Y and Gao X: Meta-analysis of the association between MALAT1 rs619586 A>G polymorphism and cancer risk. J Int Med Res 48: 300060520941969, 2020.

45. Zhang $\mathrm{P}$, Zhou $\mathrm{H}$, Lu K, Lu Y, Wang $\mathrm{Y}$ and Feng $\mathrm{T}$ : Exosome-mediated delivery of MALAT1 induces cell proliferation in breast cancer. Onco Targets Ther 11: 291-299, 2018.

46. Wang Y, Zhang Y, Hu K, Qiu J, Hu Y, Zhou M and Zhang S: Elevated long noncoding RNA MALAT-1 expression is predictive of poor prognosis in patients with breast cancer: A meta-analysis. Biosci Rep 40: BSR20200215, 2020.

47. Stone JK, Kim JH, Vukadin L, Richard A, Giannini HK, Lim SS Tan M and Ahn EE: Hypoxia induces cancer cell-specific chromatin interactions and increases MALAT1 expression in breast cancer cells. J Biol Chem 294: 11213-11224, 2019.

48. Zheng L, Zhang Y, Fu Y, Gong H, Guo J, Wu K, Jia Q and Ding X: Long non-coding RNA MALAT1 regulates BLCAP mRNA expression through binding to miR-339-5p and promotes poor prognosis in breast cancer. Biosci Rep 39: BSR20181284, 2019.

49. Fattahi Dolatabadi N, Dehghani A, Shahand E, Yazdanshenas M, Tabatabaeian H, Zamani A, Azadeh M and Ghaedi K: The interaction between MALAT1 target, miR-143-3p, and RALGAPA2 is affected by functional SNP rs3827693 in breast cancer. Hum Cell 33: 1229-1239, 2020

50. Gomes CP, Nóbrega-Pereira S, Domingues-Silva B, Rebelo K, Alves-Vale C, Marinho SP, Carvalho T, Dias S and Bernardes de Jesus B: An antisense transcript mediates MALAT1 response in human breast cancer. BMC Cancer 19: 771, 2019.

51. Wu Y, Sarkissyan M, Ogah O, Kim J and Vadgama JV: Expression of MALAT1 promotes trastuzumab resistance in HER2 overexpressing breast cancers. Cancers (Basel) 12: 1918 , 2020.

52. Huang XJ, Xia Y, He GF, Zheng LL, Cai YP, Yin Y and Wu Q: MALAT1 promotes angiogenesis of breast cancer. Oncol Rep 40: 2683-2689, 2018

53. Kim J, Piao HL, Kim BJ, Yao F, Han Z, Wang Y, Xiao Z, Siverly AN, Lawhon SE, Ton BN, et al: Long noncoding RNA MALAT1 suppresses breast cancer metastasis. Nat Genet 50: $1705-1715,2018$
54. Kwok ZH, Roche V, Chew XH, Fadieieva A and Tay Y: A non-canonical tumor suppressive role for the long non-coding RNA MALAT1 in colon and breast cancers. Int J Cancer 143: 668-678, 2018.

55. Eastlack SC, Dong S, Mo YY and Alahari SK: Expression of long noncoding RNA MALAT1 correlates with increased levels of Nischarin and inhibits oncogenic cell functions in breast cancer. PLoS One 13: e0198945, 2018.

56. Arun G and Spector DL: MALAT1 long non-coding RNA and breast cancer. RNA Biol 16: 860-863, 2019.

57. Liu SP, Yang JX, Cao DY and Shen K: Identification of differentially expressed long non-coding RNAs in human ovarian cancer cells with different metastatic potentials. Cancer Biol Med 10: 138-141, 2013.

58. Hosseini ES, Meryet-Figuiere M, Sabzalipoor H, Kashani HH, Nikzad H and Asemi Z: Dysregulated expression of long noncoding RNAs in gynecologic cancers. Mol Cancer 16: 107, 2017.

59. Sun Q, Li Q and Xie F: LncRNA-MALAT1 regulates proliferation and apoptosis of ovarian cancer cells by targeting miR-503-5p. Onco Targets Ther 12: 6297-6307, 2019.

60. Khan MA, Chen HC, Zhang D and Fu J: Twist: A molecular target in cancer therapeutics. Tumour Biol 34: 2497-2506, 2013.

61. Tania M, Khan MA and Fu J: Epithelial to mesenchymal transition inducing transcription factors and metastatic cancer. Tumour Biol 35: 7335-7342, 2014.

62. Jin Y, Feng SJ, Qiu S, Shao N and Zheng JH: LncRNA MALAT1 promotes proliferation and metastasis in epithelial ovarian cancer via the PI3K-AKT pathway. Eur Rev Med Pharmacol Sci 21: 3176-3184, 2017

63. Guo C, Wang X, Chen LP, Li M, Li M, Hu YH, Ding WH and Wang X: Long non-coding RNA MALAT1 regulates ovarian cancer cell proliferation, migration and apoptosis through Wnt $/ \beta$-catenin signaling pathway. Eur Rev Med Pharmacol Sci 22: 3703-3712, 2018.

64. Wang ZM, Wan XH, Sang GY, Zhao JD, Zhu QY and Wang DM: miR-15a-5p suppresses endometrial cancer cell growth via Wnt $/ \beta$-catenin signaling pathway by inhibiting WNT3A. Eur Rev Med Pharmacol Sci 21: 4810-4818, 2017.

65. Qiu JJ, Lin XJ, Tang XY, Zheng TT, Lin YY and Hua KQ: Exosomal metastasis-associated lung adenocarcinoma transcript 1 promotes angiogenesis and predicts poor prognosis in epithelial ovarian cancer. Int J Biol Sci 14: 1960-1973, 2018.

66. Zhou Y, Xu X, Lv H, Wen Q, Li J, Tan L, Li J and Sheng X: The long noncoding RNA MALAT-1 is highly expressed in ovarian cancer and induces cell growth and migration. PLoS One 11: e0155250, 2016.

67. Yang L, Bai HS, Deng Y and Fan L: High MALAT1 expression predicts a poor prognosis of cervical cancer and promotes cancer cell growth and invasion. Eur Rev Med Pharmacol Sci 19: 3187-3193, 2015.

68. Jiang Y, Li Y, Fang S, Jiang B, Qin C, Xie P, Zhou G and Li G: The role of MALAT1 correlates with HPV in cervical cancer. Oncol Lett 7: 2135-2141, 2014.

69. Lu H, He Y, Lin L, Qi Z, Ma L, Li L and Su Y: Long non-coding RNA MALAT1 modulates radiosensitivity of HR-HPV+ cervical cancer via sponging miR-145. Tumour Biol 37: 1683-1691, 2016.

70. Han X, Wang Q, Wang Y, Hu B, Dong X, Zhang H and Wang W: Long non-coding RNA metastasis-associated lung adenocarcinoma transcript $1 /$ microRNA-202-3p/periostin axis modulates invasion and epithelial-mesenchymal transition in human cervical cancer. J Cell Physiol 234: 14170-14180, 2019.

71. Wang N, Hou MS, Zhan Y, Shen XB and Xue HY: MALAT1 promotes cisplatin resistance in cervical cancer by activating the PI3K/AKT pathway. Eur Rev Med Pharmacol Sci 22: 7653-7659, 2018.

72. Zhao Y, Yang Y, Trovik J, Sun K, Zhou L, Jiang P, Lau TS, Hoivik EA, Salvesen HB, Sun H and Wang H: A novel wnt regulatory axis in endometrioid endometrial cancer. Cancer Res 74: 5103-5117, 2014

73. Chen G, Zhang M, Liang Z, Chen S, Chen F, Zhu J, Zhao M, He J, Hua W and Duan P: Association of polymorphisms in MALAT1 with the risk of endometrial cancer in Southern Chinese women. J Clin Lab Anal 34: e23146, 2020.

74. Cao X, Zhao R, Chen Q, Zhao Y, Zhang B, Zhang Y, Yu J, Han G, Cao W, Li J and Chen X: MALAT1 might be a predictive marker of poor prognosis in patients who underwent radical resection of middle thoracic esophageal squamous cell carcinoma. Cancer Biomark 15: 717-723, 2015. 
75. Liu R, Li J, Lai Y, Liao Y, Liu R and Qiu W: Hsa-miR-1 suppresses breast cancer development by down-regulating K-ras and long non-coding RNA MALAT1. Int J Biol Macromol 81: 491-497, 2015

76. Arun G, Diermeier S, Akerman M, Chang KC, Wilkinson JE, Hearn S, Kim Y, MacLeod AR, Krainer AR, Norton L, et al: Differentiation of mammary tumors and reduction in metastasis upon Malat1 lncRNA loss. Genes Dev 30: 34-51, 2016.

77. Gordon MA, Babbs B, Cochrane DR, Bitler BG and Richer JK: The long non-coding RNA MALAT1 promotes ovarian cancer progression by regulating $\mathrm{RBFOX} 2$-mediated alternative splicing. Mol Carcinog 58: 196-205, 2019.

78. Bai L, Wang A, Zhang Y, Xu X and Zhang X: Knockdown of MALAT1 enhances chemosensitivity of ovarian cancer cells to cisplatin through inhibiting the Notch1 signaling pathway. Exp Cell Res 366: 161-171, 2018.

79. Worku T, Bhattarai D, Ayers D, Wang K, Wang C, Rehman ZU, Talpur HS and Yang L: Long non-coding RNAs: The new horizon of gene regulation in ovarian cancer. Cell Physiol Biochem 44: 948-966, 2017.

80. Pa M, Naizaer G, Seyiti A and Kuerbang G: Long noncoding RNA MALAT1 functions as a sponge of MiR-200c in ovarian cancer. Oncol Res: Sep 11, 2017 (Epub ahead of print).

81. Lei R, Xue M, Zhang L and Lin Z: Long noncoding RNA MALAT1-regulated microRNA 506 modulates ovarian cancer growth by targeting iASPP. Onco Targets Ther 10: 35-46, 2016.

82. Zhu P, Wang FQ and Li QR: Correlation study between long non-coding RNA MALAT1 and radiotherapy efficiency on cervical carcinoma and generation of radiotherapy resistant model of cancer. Eur Rev Med Pharmacol Sci 22: 5140-5148, 2018.
83. Sun R, Qin C, Jiang B, Fang S, Pan X, Peng L, Liu Z, Li W, Li Y and Li G: Down-regulation of MALAT1 inhibits cervical cancer cell invasion and metastasis by inhibition of epithelialmesenchymal transition. Mol Biosyst 12: 952-962, 2016.

84. Xia C, Liang S, He Z, Zhu X, Chen R and Chen J: Metformin, a first-line drug for type 2 diabetes mellitus, disrupts the MALAT1/miR-142-3p sponge to decrease invasion and migration in cervical cancer cells. Eur J Pharmacol 830: 59-67, 2018.

85. Li Q, Zhang C, Chen R, Xiong H, Qiu F, Liu S, Zhang M, Wang F, Wang Y, Zhou X, et al: Disrupting MALAT1/miR-200c sponge decreases invasion and migration in endometrioid endometrial carcinoma. Cancer Lett 383: 28-40, 2016.

86. Tian X and Xu G: Clinical value of lncRNA MALAT1 as a prognostic marker in human cancer: Systematic review and meta-analysis. BMJ Open 5: e008653, 2015.

87. Du Z, Fei T, Verhaak RG, Su Z, Zhang Y, Brown M, Chen Y and Liu XS: Integrative genomic analyses reveal clinically relevant long noncoding RNAs in human cancer. Nat Struct Mol Biol 20: 908-913, 2013.

88. Liao H, Chen Q and Xiao J: Reflections on the role of Malat1 in gynecological cancer. Cancer Manag Res 12: 13489-13500, 2020 .

89. Sun Y and Ma L: New insights into long non-coding RNA MALAT1 in cancer and metastasis. Cancers (Basel) 11: 216, 2019.

90. Amodio N, Raimondi L, Juli G, Stamato MA, Caracciolo D, Tagliaferri P and Tassone P: MALAT1: A druggable long non-coding RNA for targeted anti-cancer approaches. J Hematol Oncol 11: 63, 2018. 